ISSN: 2162-3104 Print/ ISSN: 2166-3750 Online

Volume 7, Issue 2 (2017), pp. 188-203

(C) Journal of International Students

http://jistudents.org/

\title{
The Role of Attachment, Travel Experiences and English Proficiency in International Students' Acculturative Stress and Depressive Symptoms
}

\author{
Iskra Smiljanic \\ Department of Psychology, Kings County Hospital Center, USA
}

\begin{abstract}
This study examined the relationship between attachment, travel experiences, and English proficiency and international students' acculturative stress and depressive symptoms. A total of 91 graduate international students completed online surveys. Pearson correlations showed that both attachment anxiety and avoidance were positively correlated with depressive symptoms, while only attachment anxiety was positively correlated with acculturative stress. Acculturative stress was significantly higher for those participants who never traveled abroad prior to moving to the US. Additionally, lower scores on the speaking section of the TOEFL exam were related to more acculturative stress. Implications for outreach, counseling, and future research are discussed.
\end{abstract}

Keywords: international students, attachment, acculturative stress, depression, English

Each year the US attracts a large number of international students. In the 2013/14 academic year 886,052 international students were enrolled at US colleges and universities, which was an $8 \%$ increase from the previous academic year and a record high (Institute of International Education, 2014). While transitioning and adjusting to college is a time of multiple stressors and emotional turmoil for many college students (Rodgers \& Tennison, 2009), it was reported that international students experience more adjustment difficulties than their American peers (Sandhu \& Asrabadi, 1994; Mori, 2000). International students often face the additional demands of a new culture and language, separation from their home country, a decrease in regular contact with their family, and fewer 
opportunities to speak their native language (Poyrazli \& Lopez, 2007), all which can make the adjustment to college life more stressful.

Despite the unique challenges international students encounter while studying in the US, they continue to be one of the most underserved groups in US academic institutions (Mori, 2000), partially because they tend to underutilize counseling services (Mori, 2000; Nilsson, Berkel, Flores, \& Lucas 2004). In order to better serve this growing student population, it is important to gain a better understanding of the risk factors associated with adjustment difficulties.

The purpose of this study was to gain a better understanding how attachment, previous travel experiences and English language proficiency relate to international students' acculturative stress and depressive symptoms. The results of this study provide directions for future research, outreach programs, and counseling to facilitate international students' adjustment and improve their study abroad experience.

\section{LITERATURE REVIEW}

Previous research findings indicate that secure attachment is related to greater academic, social, and emotional college student adjustment (Mattanah, Hancock, \& Brand, 2004). While individuals were initially categorized as having a particular attachment style it has been argued that the categorical conceptualization of attachment is too simplistic and does not allow for the exploration of more nuanced differences between individuals within each of the attachment styles. In order to capture the nuances of people's attachment experiences, a number of researchers conceptualized attachment style categories dimensionally, with each representing a different point along a continuum (Fraley \& Waller, 1998; Brennan, Clark, \& Shaver, 1998). Brennan, Clark, and Shaver (1998) carried out a factor analysis of most of the known self-report measure of adult romantic attachment and from it derived a self-report measure that

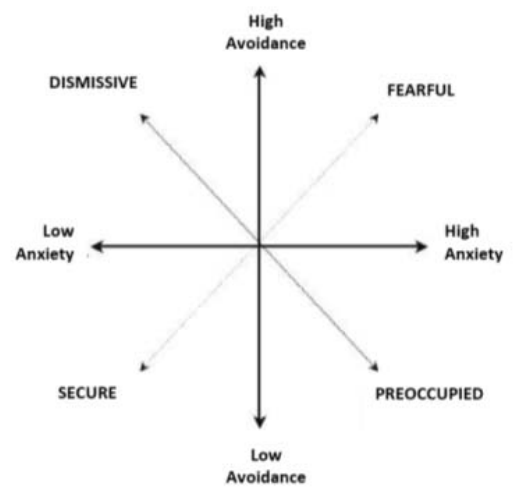
consists of two subscales, attachment-related anxiety, and attachment-related avoidance. The orthogonal anxiety and avoidance dimensions intersect and form quadrants, each of which represents a predominant attachment style (see Fig 1).

Fig 1. Two-dimensional fourcategory model of adult attachment (Brennan, Clark, \& Shaver, 1998). 
Individuals who score high on attachment anxiety (above the score where the anxiety and avoidance subscales intersect) have a tendency to worry about others' availability while those who score low on attachment anxiety (below the score where the anxiety and avoidance subscales intersect) feel more secure about others' responsiveness and availability. Individuals who score high on attachment avoidance prefer to be independent and not to rely on others, while those who score low on attachment avoidance are more comfortable being interdependent. Individuals with secure attachment score low on both anxiety and avoidance. Individuals with preoccupied attachment score high on anxiety and low on avoidance. Individuals with fearful avoidant attachment score high on anxiety and high on avoidance. Individuals with dismissive avoidant attachment score low on anxiety and high on avoidance. It was suggested that individuals vary in their attachment style across time (Waters, Merrick, Treboux, Crowell, \& Albersheim, 2000), as well as within and across relationships (Fuller \& Fincham, 1995). It was also proposed that attachment evolves throughout life depending on interpersonal experiences (Lewis, Feiring, \& Rosenthal, 2000) and that major life transitions can provide opportunities for reorganizing attachment representations.

Giuliani (2003) suggested that individuals are not only attached to people but also to places. Place attachment has been defined as an affective bond between individuals and specific environments where they feel comfortable and safe and where they prefer to be (Hernandez, Hidalgo, Salazar-Laplace, \& Hess, 2007). Places, people, and objects that are felt to be familiar can be experienced as soothing because they are more predictable and thus engender a sense of safety and reliability. In contrast, places, people, and objects that are unfamiliar can give rise to a wide range of feelings, which depending on the individual's past experiences can be both positive and/or negative. While people have the need for predictability, stability, and continuity, we also have a need for novelty and change. Throughout our lives, we are trying to balance this dynamic and go through both phases of change and growth and phases of equilibrium. Growing up, we learn to alternate between those two needs, for example like infants who explore the environment but frequently reference back and seek out their parents when they need comfort. As development proceeds, some individuals might eventually come to prefer one need over the other. Hence, while some people favor exploration and the excitement and uncertainty that go along with it, others favor the familiar over the unfamiliar -- the predictable over the unpredictable; those individuals prefer to give up some of their freedom in order to maintain a sense of stability. A person can tolerate only a certain amount of separation from familiar objects, people, and places before experiencing emotional, cognitive, somatic, and behavioral reactions, which are colloquially referred to as 
homesickness (Vingerhoets, 2005). Kenny (1987) conceptualized going abroad as the "Strange Situation" because it frequently separates students from their attachment objects and challenges their adaptive and coping mechanisms while they are trying to navigate new environments, stay connected to their old attachment objects, and establish new relationships.

As mentioned before international students are frequently faced with a foreign language. Previous research showed that difficulties with the host country's language are related to acculturative stress and depression (Yeh \& Inose, 2003; Poyrazli, Kavanaugh, Baker, \& Al-Timimi, 2004; Sumer, Poyrazli, \& Grahame, 2008) and can contribute to various issues in academic and social settings. For example, difficulties with English can negatively impact international students' academic performance, including writing papers, oral presentations, and participation in class, all which may cause stress and negatively impact self-esteem (Mori, 2000). Furthermore, language barriers may negatively impact international students' ability to socialize and establish friendships with their American peers (Mori 2000), which in turn might lead to a smaller support network and increased acculturative stress (Poyrazli et al., 2004).

Based on an extensive literature review this study proposed the following hypotheses:

$\mathrm{H}_{1}$ : Participants who score high on attachment anxiety and avoidance and thus are more likely to have insecure attachment tendencies report higher levels of acculturative stress and more depressive symptoms than participants who score low on attachment anxiety and avoidance.

$\mathrm{H}_{2}$ : The level of acculturative stress is positively correlated with the level of depressive symptoms.

$\mathrm{H}_{3}$ : Traveling abroad prior to studying abroad is negatively correlated with the level of acculturative stress.

$\mathrm{H}_{4}$ : Better English proficiency prior to studying abroad is negatively correlated with the level of acculturative stress at the time of the study.

\section{RESEARCH METHOD}

This study was approved by The City University of New York institutional review board (IRB) for research on human subjects. Data were collected via an online survey. Participants were recruited through emails that were sent to university-based International Student Offices and international student organizations in New York City and Chicago, which were asked to distribute the email through their email list serve. The recruitment email briefly explained the study and contained a link and the password to the online survey. Once participants signed into the online survey they were presented with the informed consent form and asked to electronically sign it 
before filling out the survey. It took participants approximately 25 minutes to complete the online survey. Upon completing the online survey and confirming their date of arrival to the US, participants were emailed a $\$ 10$ Amazon gift card as remuneration. Only complete entries were used for the research. A complete entry consisted of a filled out online survey with a signed consent form and confirmed date of arrival to the US. Out of 176 entries, a total of 91 entries met the above inclusion criteria and were used for this study.

\section{Participants}

Participants were 91 international graduate students who were enrolled in a US graduate school program at the time of the data collection. This study recruited only graduate students because as a group they were likely to be more homogenous than undergraduates, to have consolidated a firmer sense of identity, and to experience less developmental challenges which could have impacted their adjustment to the US. Participants consisted of 46 females (50.5\%) and 45 males (49.5\%). Out of the 91 participants, a total of 89 reported their age. Participants' ages ranged from 22 to 45 years, with a mean age of $28.88(\mathrm{SD}=3.93)$. Out of the 91 participants, 42 participants (46\%) identified themselves as Asian, 35 participants $(38 \%)$ as White, 6 participants $(7 \%)$ as Latino, and 8 participants (9\%) as "Other." Participants were grouped into the following geographic regions: 35 participants from Europe (39\%), 16 participants from China (18\%), 14 participants from India (16\%), 10 participants from other Asian countries (11\%), 4 participants from Middle East (4\%), 4 participants from South America (4\%), 3 participants from Canada (3\%), 3 participants from Latin America (3\%), 1 participant from Australia (1\%), and 1 participant from Caribbean (1\%). The majority of participants (87\%) reported English was their second language. Out of the 72 participants who reported the graduate degree they seek, 10 were master's students (14\%) and 62 participants were doctoral students (86\%). Participants' length of stay in the US ranged from .51 to 5.81 years, with an average of 2.91 years.

\section{Measures and Instruments}

Demographic questionnaire. Participants completed a demographic questionnaire on background information including age, country of origin, date of arrival to the US, travel experiences, and TOEFL scores. TOEFL is an English-language test most US universities require international students to take prior to applying for admission to assess their English proficiency. 
Depression. Depressive symptoms were assessed with the Center for Epidemiological Studies Depression Scale (CES-D; Radloff; 1977). This is a 20 -item self-report measure asking participants to rate symptoms of depression for the past week on a scale from 0 (meaning less than 1 day) to 3 (5-7 days). Total scores can range from 0 to 60. It takes about 5 to 10 minutes to complete this questionnaire. The reported internal consistency, as measured by Cronbach's alpha, is high across a variety of populations (around .85 in community samples and .90 in psychiatric samples). Testretest reliability studies ranging from two to eight weeks show moderate correlations $(\mathrm{r}=.51-.67)$, which is desirable for a test of symptoms that are expected to show change over time.

Acculturative Stress. Acculturative Stress was measured using the Acculturative Stress Scale for International Students (ASSIS; Sandhu \& Asrabadi, 1994). The ASSIS is a 36-item, 5-point (1 strongly disagree, 3 unsure, 5 strongly agree), Likert-type scale that assesses adjustment-related issues of international students. It consists of seven subscales - Perceived Discrimination (8 items), Homesickness (4 items), Perceived Hate (5 items), Fear (4 items), Stress Due to Change/Culture Shock (3 items), Guilt (2 items), and Nonspecific Concerns (10 items). The total scores for this scale can range from 36 to 180 , with higher scores indicating higher levels of acculturative stress. Validity was supported by a factor analysis and reliability was provided by Cronbach's coefficient alphas ranging from .87 to .95 for the total score of the ASSIS (Poyrazli et al., 2004; Sandhu \& Asrabadi, 1994; Yeh \& Inose, 2003).

Attachment. Attachment anxiety and avoidance were determined with the Experiences in Close Relationships Inventory (ECR; Brennan, Clark, \& Shaver; 1998), which is a 36-item self-report measure that assesses individuals' experiences in relationships and their attachment style. The items were derived from a factor analysis of most of the existing self-report measures of adult romantic attachment. The measure consists of two separate scales, avoidance and anxiety. The anxiety subscale (18 items, such as "I worry about being abandoned") measures the excessive need for approval from others and fear of interpersonal rejection or abandonment. The avoidance subscale (18 items, such as "I prefer not to show a partner how I feel deep down") taps into an excessive need for self-reliance and fear of interpersonal closeness. Items are rated on a 7-point Likert scale, ranging from 1 (strongly disagree) to 7 (strongly agree). The reported internal consistency, as measured by Cronbach's alpha, is .94 for the avoidance subscale and .91 for the anxiety subscale. 


\section{RESULTS}

\section{Correlation between attachment anxiety/avoidance, acculturative stress, and depressive symptoms}

Pearson correlation was used as a metric to determine the relationship between variables discussed attachment anxiety/ avoidance, acculturative stress and depressive symptoms. All statistical analyses used an alpha level of .05 and two-tailed tests unless otherwise stated. Ninetyone participants completed the Experiences in Close Relationships Inventory (ECR). 57 participants (63\%) scored as securely attached, 23 participants $(25 \%)$ as preoccupied, 8 participants $(9 \%)$ as dismissive, and 3 participants (3\%) as fearful. There was a statistically significant positive correlation between the attachment anxiety subscale of the ECR and the level of acculturative stress as measured by the ASSIS $(r(89)=.46$, $p<$ .01 ), indicating that a higher level of attachment anxiety was related to more acculturative stress. There was not a statistically significant correlation between the attachment avoidance subscale of the ECR and the level of acculturative stress $(r(89)=.16, p=.12)$, indicating that the level of attachment avoidance was not significantly related to the level of acculturative stress. The relationship between the attachment anxiety/avoidance subscales and acculturative stress is illustrated in the scatterplots in Figure 2.
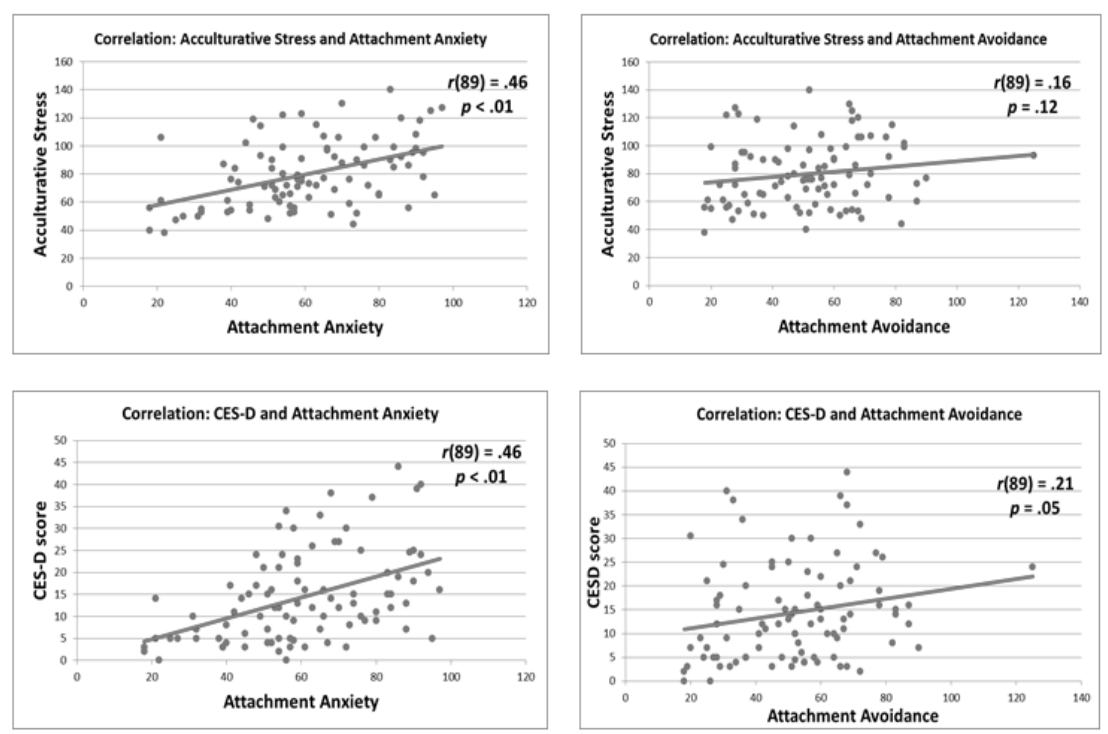

Fig 2. Correlations between attachment anxiety/avoidance, ASSIS and CES-D scores. 
There was a statistically significant positive correlation between the attachment anxiety subscale of the ECR and the number of depressive symptoms on CES-D $(\mathrm{r}(89)=.46, \mathrm{p}<.01)$, as well as the avoidance subscale of the ECR and the number of depressive symptoms on CES-D ( $\mathrm{r}(89)=.21, \mathrm{p}=.05)$. These results indicated higher attachment anxiety and avoidance were related to more depressive symptoms. The relationship between the attachment anxiety/ avoidance subscales and depressive symptoms is also illustrated in the scatterplots in Figure 2. There was also a statistically significant positive correlation between the level of acculturative stress on the ASSIS and number of depressive symptoms on CES-D $(\mathrm{r}(89)=.59, \mathrm{p} \leq .01)$, indicating that a higher level of acculturative stress was related to more depressive symptoms.

\section{Variance analysis of mean acculturative stress for groups with different travel experiences}

A one-way ANOVA was conducted to compare all 91 participants who were divided into three groups based on their travel experiences prior to studying abroad. The first group consisted of 23 participants (25\%) who never traveled abroad before moving to the US. The second group consisted of 27 participants (30\%) who traveled prior to moving to the US, yet their travels excluded the US. The third group consisted of 41 participants (45\%) who visited the US before moving to the US for their studies. There was a statistically significant difference in the mean acculturative stress level among these groups as determined by a one-way ANOVA, $\mathrm{F}(2,88)=5.92$, $\mathrm{p} \leq .004$. As can be seen in Table 1 and Figure 3, a Tukey post-hoc test indicated that acculturative stress was significantly higher for participants who never traveled abroad prior to moving to the US $(M=93.78 \pm 5.02$, $\mathrm{SD}=24.09)$ as compared to those who visited the US before studying abroad $(\mathrm{M}=75.02 \pm 3.27, \mathrm{SD}=20.95)$ and those who traveled but whose travels excluded the US $(\mathrm{M}=75.30 \pm 4.49, \mathrm{SD}=23.32)$. There was no statistically significant difference in the level of acculturative stress between participants who traveled to the US before studying abroad and those who traveled abroad but whose travels did not include the US.

\section{Table 1: p-values for Multiple Comparisons of Mean ASSIS Scores for Groups with Different Previous Travel Experiences $(N=91)$}

Previous travel experience

No travel

Travel, no US

Travel, no US

$.013 *$

Travel to US

$.005 *$

.999

$* p<.05$ 


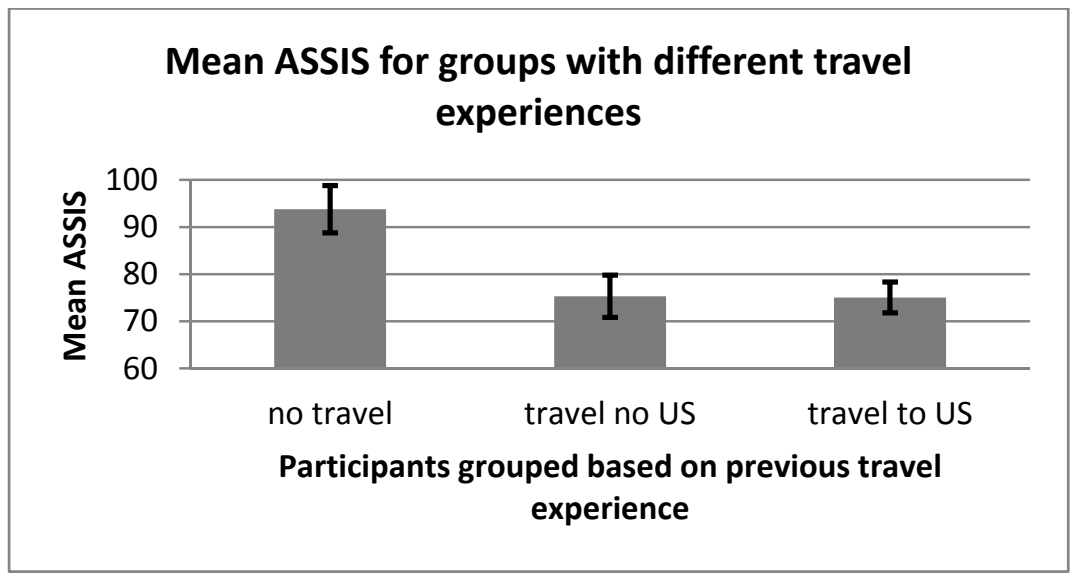

Fig 3. Average acculturative stress (ASSIS) for groups with different travel experiences with standard error bars.

\section{Correlation of TOEFL scores and acculturative stress}

Forty-one participants reported their TOEFL scores. Other students either did not take the TOEFL as English was their first language, took a similar but different test assessing their English skills, or reportedly could not recall their scores. As it was expected that there would be a negative correlation between the participants' TOEFL scores and the level of acculturative stress on the ASSIS, one-tailed Pearson correlations with an alpha level of .05 were used. Results showed that higher scores on the speaking section of the TOEFL exam were negatively correlated with the level of acculturative stress, $r(39)=-.26, p=.049$. There was no significant relationship between acculturative stress and the scores on the other subtests of the TOEFL, namely the reading score $(r(39)=.07, p=.33)$, the listening scores $(r(39)=-.09, p=.28)$, and the writing score $(r(39)=-$ $0.03, p=.44)$. A second analysis was conducted that only included scores for 33 participants who stayed in the US for no longer than three years, because those who stayed in the US longer presumably already improved their English to the point that there would not be a strong correlation between their initial English skills and acculturative stress at the time of this study. The results indicated that there was a negative correlation between the English speaking skills and the level of acculturative stress, $r(31)=-.30, p=.046$.

\section{DISCUSSION AND CONCLUSIONS}

The main purpose of the present study was to investigate the relationship between attachment style, travel experiences prior to studying abroad, 
English skills and international students' acculturative stress, and depressive symptoms. Out of 91 participants, 63\% scored as securely attached, $25 \%$ as preoccupied, $9 \%$ as dismissive, and $3 \%$ as fearful. Given the small sample of this study, there were not enough participants in the fearful group to allow for meaningful comparisons of acculturative stress and depressive symptoms between the different attachment style groups. Therefore, attachment was measured as a continuous rather than categorical variable. As expected, higher levels of attachment anxiety were related to higher levels of acculturative stress. Higher levels of attachment anxiety and avoidance were related to more depressive symptoms. These results are consistent with previous literature on the impact of attachment style on college students' adjustment to the transition from high school to college. For example, it was found that insecurely attached late adolescents reported greater depression, anxiety, and worry when compared to securely attached students (Vivona, 2000) and that secure attachment was correlated with positive college student adjustment (Mattanah, Hancock, \& Brand, 2004).

Interestingly the results of this study showed that higher levels of attachment avoidance were not related to higher levels of acculturative stress despite being related to more depressive symptoms and despite the positive correlation between depressive symptoms and acculturative stress. A possible explanation for this finding could be that participants who scored high on attachment avoidance were less aware, less self-disclosing, or minimized their level of acculturative stress and homesickness, as it would imply they are dependent on others, which may be too threatening and destabilizing for their self-concept. Alternatively, Fraley and Shaver (1997) found that highly dismissing individuals are less affected by the loss of a loved one, not only because they actively work to inhibit the activation of their attachment systems but also because they are less emotionally invested in relationships. Because their sense of self is less intertwined with relationships, the loss of or change in former relationships might not be as distressing for people with a dismissive-avoidant attachment style tendency as compared to people with a preoccupied attachment style tendency. It has been suggested that dismissive attachment has an adaptive function and may serve as a protective factor. Fraley and Bonanno (2004) found that attachment-related anxiety is correlated with elevated symptoms of grief and distress, whereas attachment-related avoidance is not. In one study they tracked individuals' reactions to the loss of a loved one and found that dismissing-avoidant individuals appeared less distressed than others and did not exhibit a reappearance of distress-related symptoms at a later point in time. Similar to secure participants, dismissive-avoidant individuals showed a resilient response to the loss of a loved one. Consequently, they suggested that adults with higher attachment avoidance may be less vulnerable when faced with interpersonal loss than has been suggested in the past. 
While this study found a statistically significant positive correlation between the number of depressive symptoms and the level of acculturative stress, it is important to keep in mind that this relationship is correlational, not causal. For example, it is possible that homesickness or perceived discrimination, both of which are subscales on the ASSIS, contribute to depressive symptoms. At the same time, it is also possible that depressed mood negatively impacts one's perception of the studying abroad experience. Thus, further research is needed to investigate the directionality of the relationship between depressive symptoms and acculturative stress.

As expected, the level of acculturative stress was significantly lower for participants who traveled outside of their home country before studying abroad as compared to participants who never traveled abroad prior to moving to the US. There was no statistically significant difference in the mean level of acculturative stress between participants who traveled to the US before studying abroad and those who traveled but whose travels did not include the US. Traveling prior to going abroad may have allowed individuals to practice separating from familiar people, environments and objects, and adjusting to unfamiliar environments. It might have also offered the opportunity to explore how to bridge the physical distance to their loved ones and form new relationships. Through this process, traveling may have provided individuals with confidence that if they have been abroad before, they can do it again. It is reasonable to think that acculturative stress could be moderated by the quality of previous travel experiences. For example, if previous travel experiences were negative then individuals might worry that they will have a negative experience again and might experience more difficulties adjusting to going abroad. It is likely that socioeconomic status also plays a moderating role. Higher socioeconomic status may offer individuals more opportunities not only to travel before studying abroad but also to visit their home country once they move abroad.

As predicted, higher TOEFL scores, specifically on the speaking section of the TOEFL exam, were negatively correlated with the level of acculturative stress. This correlation was slightly stronger when only participants who stayed in the US up to three years were included. The relationship between English skills and acculturative stress might be stronger for those who stayed in the US up to three years because the ones who stayed in the US longer might have already improved their English to the point that their initial English skills may not be as strongly related to their acculturative stress level at the time of this study. There was no significant relationship between acculturative stress and participants' scores on the other TOEFL subtests, including the reading, listening, and writing subtests. One possible explanation for this finding may be that the speaking skills might be more relevant for the adjustment process as compared to reading or writing skills because speaking more directly impacts 
international students in their everyday life. Better English skills make it easier for international students to navigate the new environment and interact with others in the new environment, all which may contribute to better self-esteem and adjustment (Yeh \& Inose, 2003). Better English speaking skills may also make international students feel less embarrassed or self-conscious about their language skills or accent, which in turn could make them feel more comfortable to interact with others and thereby increase their social support network. Conversely, difficulties with speaking English might increase stress and contribute to adjustment difficulties and social isolation.

Yeh and Inose (2003) emphasized that language is linked with culture and found that students from Asia and Central/Latin America experienced more acculturative stress than students from Europe. They hypothesized that European international students may encounter less racism and discrimination than students from Asia, Africa, and Latin America. Their sample of European students was all White racially, which may have allowed those students to blend more into the dominant racial group in the United States. They also suggested that since European and American cultural values are similar, European international students may experience fewer differences in values and the cultural patterns of behavior, all which may facilitate their adjustment to the US. However, it was not clear from their study to what extent English skills were related to European international students' lower levels of acculturative stress. As there is not much literature on the relationship between country/culture of origin, race, perceived discrimination, language and acculturative stress, this could be an area for future investigation.

\section{STUDY LIMITATIONS}

The sample for this study was very specific and results need to be interpreted with this in mind. First, all participants were graduate students. Given different developmental tasks, one should be cautious when generalizing this study's findings to other groups of international students, such as undergraduate students. Furthermore, given the temporary legal status of international students in the US, it is possible that there are specific periods of increased stress. One such period could be, for example, graduation when international students are faced with the decision whether to stay in the US or return to their home country. As graduation approaches, international students may experience the additional stressor of finding a way to stay in the US legally after graduation, which may negatively influence their mood and thus their experience of living in the US. This is also important to keep in mind for this present study, because it may have impacted some participants' scores on the CES-D, ASSIS, and ECR. 
Second, the participants for this study came from major metropolitan areas, mostly New York City and Chicago, both of which are ethnically and culturally diverse. Thus, the participants in this study likely had some shared experiences. However, recruiting international students from less diverse geographical areas in the US may yield different results. For example, international students who do not belong to the dominant ethnic or racial group in the area where they live may experience more discrimination and difficulty establishing a support network. These students may also have less opportunity to interact with people from their own background or obtain familiar and comforting objects, such as familiar food, all of which could make their adjustment to the US more difficult and increase their feelings of homesickness.

Third, attachment was assessed using a self-report measure. Selfreport measures, such as the ECR, ask adults about their typical ways of forming and being in relationships. Thus, these only measure aspects of internal working models that are within individuals' conscious awareness. As such, central aspects of attachment theory, such as the wish for closeness and autonomy, may be distorted by defenses (Westen, 1991). For example, individuals with high attachment avoidance may be uncomfortable revealing or getting in touch with experiences that would imply that they depend on others and thus threaten their self-concept (Main, Kaplan, \& Cassidy, 1985). This is important to keep in mind as it could have impacted participants' responses on the self-report measures and thereby the results of this study.

\section{DIRECTIONS FOR FUTURE RESEARCH}

Rather than relying on self-report measures, future studies could use a more implicit measure of attachment, such as the AAI, and compare participants' answers on that measure to their responses on the acculturative stress measure. This could control for defenses that might otherwise skew their attachment classification and thus the relationship between attachment and acculturative stress. Furthermore, future studies could follow international students throughout the course of their studies and explore what personal or community supports help them effectively manage their study abroad experience.

While this sample was too small for the purpose, future studies could continue to assess the relationship between English skills during the first year of studying abroad and acculturative stress. It is probable that language skills play a crucial role in the adjustment process in the beginning when students are still new to the environment and are trying to establish relationships. For graduate students, English skills might be particularly important because they frequently function as teaching assistants and as such are required to teach and interact with other students 
in English. If a teaching assistant struggles with English, it could negatively impact their mood, self-esteem, and professional identity and lead to adjustment difficulties. Finally, further research is needed to study the experiences of international students from cultures that are greatly different than the host country, for example, more conservative than the dominant US culture, to better understand how such differences may impact adjustment.

\section{IMPLICATIONS}

Despite its limitations, this study identified factors which impact international students' adjustment and have implications for outreach programs and counseling. The findings of this study emphasize the importance of providing international students with support to help them adjust to the US. All participants in this study were enrolled in a university. The simple fact of belonging to a university may foster resilience in international students and reduce their feelings of isolation by providing them with opportunities for social interactions and a sense of belonging. Universities could use the milieu to facilitate international students' adjustment. For example, international students could be paired up with more senior international students who could function as their mentors. Additionally, they could be paired up with American peers who could introduce them to the US culture and customs, thereby also facilitating the development of cross-cultural friendships. Jacob and Greggo (2001) suggested pairing up graduate counseling students and international students for a semester to provide international students with additional social support and an opportunity to get to know the US culture, while also providing counselors-in-training with a multicultural experience to refine their cultural sensitivity.

As previous research found that international students underutilize counseling services (Mori, 2000), it may be more effective to develop alternatives to traditional counseling to increase international students' knowledge about potential adjustment challenges, coping skills, and resources. This would provide international students with additional support and a platform from which they could develop a better understanding of their own adjustment experiences and establish initial friendships. For example, universities could offer informal weekly groups for international students in which they could socialize and share their experiences of living abroad. Such groups could be facilitated by counseling center staff who could collaborate with the International Students Office to address specific concerns international students have. Counseling centers could offer workshops during particularly difficult times in an international student's school career, such as the beginning of 
their studies or graduation. There could also be web-based forums where students could share articles about their experiences on a monthly basis.

Finally, the results of this study can be applied to individual and group counseling. Counselors could explore international students' adjustment through the lens of attachment theory by discussing their attachment tendencies, reactions to past separations, and self-concept and help them identify how those relate to their adjustment experiences in the US. In this context, counselors could help them identify coping skills during previous separations and apply those skills to their life in the US. Furthermore, international students could develop a deeper understanding of their adjustment experiences by exploring with a counselor the similarities and differences between their culture of origin and the US culture and how these impact their adjustment and expectation of their life in the US.

\section{REFERENCES}

Brennan, K.A., Clark, C.L., \& Shaver, P.R. (1998). Self-report measurement of adult romantic attachment: An integrative overview. In J.A. Simpson \& W.S. Rholes (Eds.), Attachment Theory and Close Relationships (pp. 46-76). New York: Guilford Press.

Fraley, R. C., \& Bonanno, G. A. (2004). Attachment and loss: A test of three competing models on the association between attachment-related avoidance and adaptation to bereavement. Personality and Social Psychology Bulletin, 30, 878-890.

Fraley, R. C., \& Shaver, P. R. (1997). Adult attachment and the suppression of unwanted thoughts. Journal of Personality and Social Psychology, 73, 1080-1091.

Fraley, R.C., \& Waller, N.G. (1998). Adult attachment patterns: A test of the typological model. In J.A. Simpson \& W.S. Rholes (Eds.), Attachment Theory and Close Relationships (pp. 77-114). New York: Guilford Press.

Fuller, T.L., \& Fincham, F.D. (1995). Attachment style in married couples: Relation to current marital functioning, stability over time, and method of assessment. Personal Relationships, 2, 17-34.

Giuliani, M.V. (2003). Theory of attachment and place attachment. In M. Bonnes, T. Lee, \& M. Bonaiuto (Eds.), Psychological theories for environmental issues (pp. 137-170). Aldershot: Ashgate Publishing, Ltd.

Hernandez, B., Hidalgo, M.C., Salazar-Laplace, M.E., \& Hess, S. (2007). Place attachment and place identity in natives and non-natives. Journal of Environmental Psychology, 27, 310-319.

Institute of International Education. (2014). Open Doors Report on International Educational Exchange. Retrieved from http://www.iie.org/opendoors

Jacob, E. J., \& Greggo, J. W. (2001). Using counselor training and collaborative programming strategies in working with international students. Journal of Multicultural Counseling and Development, 29 (1), 73-84.

Kenny, M.E. (1987). The extent and function of parental attachment among first-year college students. Journal of Youth and Adolescence, 16 (1), 17-29.

Lewis, M., Feiring, C., \& Rosenthal, S. (2000). Attachment over time. Child Development, 71 (3), 707-720.

Main, M., Kaplan, N., \& Cassidy, J. (1985). Security in infancy, childhood, and adulthood: A move to the level of representation. Monographs of the Society for Research in Child Development, 50, 66-104. 
Mattanah, J.F., Hancock, G.R., \& Brand, B.L. (2004). Parental attachment, separationindividuation, and college student adjustment: A structural equation analysis of mediational effects. Journal of Counseling Psychology, 51 (2), 213-225.

Mori, S. (2000). Addressing the mental health concerns of international students. Journal of Counseling \& Development, 78 (2), 137-144.

Nilsson, J.E., Berkel, L.A., Flores, L.Y., \& Lucas, M.S. (2004). Utilization rate and presenting concerns of international students at a university counseling center: Implications for outreach programming. Journal of College Student Psychotherapy, 19 (2), 49-59.

Poyrazli, S., Kavanaugh, P.R., Baker, A., \& Al-Timimi, N. (2004). Social support and demographic correlates of acculturative stress in international students. Journal of College Counseling, 7, 73-82.

Poyrazli, S., \& Lopez, M.D. (2007). An exploratory study of perceived discrimination and homesickness: A comparison of international students and American students. The Journal of Psychology, 141 (3), 263-280.

Radloff, L. (1977). The CES-D scale: A self-report depression scale for research in the general population. Applied Psychosocial Measurement, 1, 385-401.

Rodgers, L.S., \& Tennison, L.R. (2009). A preliminary assessment of adjustment disorder among first-year college students. Archives of Psychiatric Nursing, 23 (3), 220 -230.

Sandhu, D.S., \& Asrabadi, B.R. (1994). Development of an acculturative stress scale for international students: Preliminary findings. Psychological Reports, 75, 435-448.

Sumer, S., Poyrazli, S., \& Grahame, K. (2008). Predictors of depression and anxiety among international students. Journal of Counseling \& Development, 86 (4), 429-437.

Vingerhoets, A. (2005). The homesickness concept: Questions and doubts. In A.L.M. van Tilburg \& J.J.M. Vingerhoets (Eds.), Psychological aspects of geographical moves: Homesickness and acculturation stress (pp.1-16). Amsterdam: Amsterdam U. Press.

Vivona, J.M. (2000). Parental attachment styles of late adolescents: Qualities of attachment relationships and consequences for attachment. Journal of Counseling Psychology, 47 (3), 316-329.

Waters, E., Merrick, S., Treboux, D., Crowell, J., \& Albersheim, L.(2000). Attachment Security in infancy and early adulthood: A twenty-year longitudinal study. Child Development, 71 (3), 684-689.

Westen, D. (1991). Social cognition and object relations. Psychological Bulletin, 109 (3), 429-455.

Yeh, C.J., \& Inose, M. (2003). International students' reported English fluency, social support satisfaction, and social connectedness as predictors of acculturative stress. Counseling Psychology Quarterly, 16 (1), 15-28.

Note: This study was facilitated by a Research grant for graduate students from The Graduate Center, City University of New York to Iskra Smiljanic.

ISKRA SMILJANIC, Ph.D., is a clinical psychologist who has experience working with international students in US college counseling centers. Her areas of interest include the psychological impact of immigration, attachment, and the development of prevention/intervention programs addressing the cultural adjustment process.

Email: iskrasmiljanic@gmail.com

Manuscript submitted: September 9, 2015

Manuscript Revised: January 17, 2016

Accepted for publication: February 20, 2016 